\title{
Deutsche Verfassungsgeschichtsschreibung im 20. Jahrhundert im Spannungsfeld von Politik und Wissenschaft ${ }^{1}$
}

Daß Wissenschaft keine unpolitische Angelegenheit ist, gehört zu den selbstverständlichen propädeutischen Feststellungen. Wissenschaft ist zeitgebunden, insofern eben auch politisch beeinflußbar und notwendig subjektiv. So gilt das Objektivitätsgebot historischen Forschens - das so gern mit Zitaten Leopold von Rankes belegt wird - als unerreichbares Ideal; gleichwohl besitzt es - mit allen Einschränkungen - Leitwertcharakter. Die Geschichtswissenschaft ist ebenso wie andere Fächer eingebettet in historische Bedingungen und Geschehnisse, in politische Strukturen und Prozesse. Geschichte und Lebenswelt stehen in einem unaufhebbaren Spannungsverhältnis und in einem vielgliedrigen Beziehungsgeflecht. Wissenschaftliche Forschung ist stets perspektivgebunden, verfolgt Intentionen und ist ebenso Spiegel wie Funktion der Gesellschaft.

Dieser Beitrag soll den Wirkungszusammenhang beschreiben, in dem sich ein Teil der Geschichtsschreibung unseres Jahrhunderts bewegt hat: das Spannungsfeld von Politik und Wissenschaft, das sich personen-, institutionen- und ideengeschichtlich auswirkt ${ }^{2}$. Denn Wissenschaftsverständnis und Forschungsergebnisse stehen gleichsam im Strom der realen Geschichte. Es gilt nachfolgend, wie der Staatsrechtler und Verfassungshistoriker Ernst-Wolfgang Böckenförde es einmal formuliert hat, »die Entwicklung der verfassungsgeschichtlichen Forschung als einen geschichtlich gebundenen Vorgang zu begreifen ${ }^{3}$. Die Diskontinuitäten der deutschen Geschichte im 20. Jahrhundert haben Auswirkungen auf den wissenschaftsgeschichtlichen Entwicklungsgang der Verfassungsgeschichtsschreibung gehabt. Zudem stand die Verfassungshistoriographie stets im Zusammenhang mit der aktuellen Verfassungslage. Wie aber sieht eine solche Korrelation genau aus und welche Konstellationen ruft sie hervor?

Die Hauptlinien der Entwicklung der Verfassungsgeschichtsschreibung in Deutschland werden anschließend in vier chronologischen Etappen verfolgt ${ }^{4}$. Gemäß den verfassungshistorischen Zäsuren unseres Jahrhunderts ist die Forschung vor 1918, in der Weimarer Zeit, dem Nationalsozialismus und der Nachkriegsepoche zu würdigen. Besonderes Interesse verdient dabei der Verfassungshistoriker Ernst Rudolf Huber,

1 Grundgedanken dieses Textes wurden im Bochumer Politikwissenschaftlichen Seminar bei Wilhelm Bleek, später dann im Kolloquium des Historischen Seminars der Bergischen Universität Wuppertal bei Hartwig Brandt, Hermann de Buhr, Franz Knipping und Heinrich Küppers vorgetragen. Ich danke den Teilnehmern der Veranstaltungen für Anregungen. Ulrich Sieg hat in bewährter Weise den Text durchgesehen.

2 Methodische Vorbemerkungen ähnlicher Art bei Wilhelm Bleek: Geschichte der Politikwissenschaft in Deutschland, München 2001, S. 15-28.

3 Ernst-Wolfgang Böckenförde: Die deutsche verfassungsgeschichtliche Forschung im 19. Jahrhundert. Zeitgebundene Fragestellungen und Leitbilder, Berlin 1961/2²995 (= Schriften zur Verfassungsgeschichte, 1), S. 19.

4 Ausführlich dazu meine Wuppertaler Habilitationsschrift, die sich im Druck befindet: Ewald Grothe: Zwischen Geschichte und Recht. Deutsche Verfassungsgeschichtsschreibung 1900-1970, München 2005 (= Ordnungssysteme, 16). 
dessen Person und Werk wie kaum ein anderes die Wandlungen der Verfassungsgeschichtsschreibung im 20. Jahrhundert widerspiegeln. Zuletzt werden kurz die Perspektiven der jüngsten Forschungsgeschichte aufgezeigt.

\section{Vorüberlegungen}

\section{a) Der Begriff und das Teilfach der Verfassungsgeschichte}

Was versteht man überhaupt unter Verfassungsgeschichte? Die inhaltliche Konturierung des Begriffs ist durchaus nicht eindeutig. Denn eine klare fachliche Zuordnung der Verfassungsgeschichte ist nicht möglich. Allein drei wissenschaftliche Disziplinen erheben Ansprüche auf sie: die Historiker, weil Verfassungsgeschichte als Teil der politischen, konkreter der innenpolitischen Geschichte verstanden wird; die Juristen, weil die staatliche Verfassung vor allem aus Gesetzen besteht, also juristischer »Alltagsware « par excellence; und schließlich die Politikwissenschaftler: Hans Boldt plädiert nachdrücklich für eine politik-wissenschaftliche, d.h. eine historisch arbeitende vergleichende Regierungslehre als Kernbereich der Verfassungsgeschichtsforschung ${ }^{5}$.

Welcher Begriff von Verfassungsgeschichte und Verfassungsgeschichtsschreibung liegt aber den nachfolgenden Ausführungen zugrunde? Eine verkürzende Arbeitsdefinition beschränkt sich ganz bewußt auf die politisch-juristische Dimension der Verfassung. Unter Verfassungsgeschichtsschreibung werden nachfolgend alle diejenigen historischen, juristischen und politikwissenschaftlichen Studien gefaßt, die sich mit den politisch-gesellschaftlichen Strukturen auseinandersetzen, d.h. z.B. Fragen nach der Staatsform und dem Regierungssystem, nach politischer Partizipation und Repräsentation, nach den politisch-gesellschaftlichen Institutionen und Organisationen. Es geht um Studien, die sich mit der politischen und sozialen Verfaßtheit, den Strukturen einer Gesellschaft beschäftigen - Studien, deren Terrain das »Handeln in Verfassung « ist ${ }^{6}$.

\section{b) Traditionen}

Historische Untersuchungen des 20. Jahrhunderts über die politische und soziale Ordnung, die Verfaßtheit eines Gemeinwesens, stehen in einer längeren Tradition. Den Ergebnissen von Fritz Hartung und Hans Boldt folgend ${ }^{7}$, hat die Verfassungsge-

5 Hans Boldt: Verfassungsgeschichte und vergleichende Regierungslehre. Zur Geschichte ihrer Beziehungen. In: Der Staat 24 (1985), S. 432-446. Vgl. auch ders.: Verfassungsgeschichte - Bemerkungen zur Historie einer politik-wissenschaftlichen Disziplin. In: ders., Einführung in die Verfassungsgeschichte. Zwei Abhandlungen zu ihrer Methodik und Geschichte, Düsseldorf 1984, S. 119-217, hier S. 174-185.

6 Hartwig Brandt: Verfassungsgeschichte. Standort und Probleme einer historischen Disziplin, unveröff. Ms., Marburg a.d. Lahn 1976, S. 10 ff. Ich danke sehr für die Überlassung des ungedruckten Habilitationsvortrages.

7 Fritz Hartung: Zur Entwicklung der Verfassungsgeschichtsschreibung in Deutschland, Berlin 1956 (= Sitzungsberichte der Deutschen Akademie der Wissenschaften zu Berlin. Klasse für Philosophie usw. 1956, 3); Hans Boldt: Verfassungsgeschichte - Bemerkungen zur Historie (wie Anm. 5). 
schichtsschreibung ihre Wurzeln in der älteren Reichshistorie vor 1800. Untersuchungen über das Recht vergangener Zeiten hatten zu Beginn des 19. Jahrhunderts, in den Zeiten romantischer Rückbesinnung, Konjunktur. Nun begann eine erste wissenschaftliche Aufbereitung und Vergegenwärtigung der Vergangenheit, die auf dem juristischen Sektor im Zeichen der Historischen Rechtsschule unter dem Primat der Historie stand. Die alte Reichsgeschichte wandelte sich zur Rechtsgeschichte ${ }^{8}$.

Der Kieler Historiker Georg Waitz hat 1844 wohl erstmals den Begriff »Verfassungsgeschichte « geprägt, um in einem voluminösen mehrbändigen Werk den Anfängen der deutschen Geschichte im frühen Mittelalter nachzugehen ${ }^{9}$. Während die systematisch angelegten Darstellungen der Juristen seit der Jahrhundertmitte durchgängig mit »Rechtsgeschichte« titelten, nannten sich die historischen Untersuchungen zu Kaiser und Fürsten, Kirchen- und Reichsgeschichte sowie zur Stadt- und Sozialverfassung (vornehmlich) des Mittelalters »Verfassungsgeschichte«. Eine eigentliche und eigenständige Verfassungsgeschichte der Neuzeit war dem 19. Jahrhundert unbekannt.

\section{Die versandete Innovation: von Otto Hintze zu Fritz Hartung}

Die historische Verfassungsgeschichte um 1900 stand ganz im Bann der traditionellen politischen Ereignisgeschichte. Individualitätsaxiom und der Primat der Außenpolitik prägten die Geschichtsbetrachtung im Zeichen des Historismus. Die historische Forschung war zudem überwiegend national orientiert, bisweilen geradezu germanozentrisch. Die Verfassungsgeschichtsschreibung hatte ihrerseits noch kein spezifisches Terrain erobert. Der innovativste Vertreter unter den Historikern war ohne Zweifel der langjährige Berliner Lehrstuhlinhaber (1902-1920) für Verfassungs-, Verwaltungs-, Wirtschaftsgeschichte und Politik, Otto Hintze. Seine Verfassungsgeschichtsschreibung beruhte auf Rechtsvergleich und Institutionengeschichte, ging vom Staat aus und war an staatlichem Handeln orientiert, allerdings mit einer expliziten Ausrichtung auf die vergleichenden und typologischen Aspekte. Seine Forschungen legte er in zahlreichen Aufsätzen, vor allem zur preußischen Geschichte der Frühen Neuzeit, nieder. In den zwanziger Jahren suchte er sie methodisch in Anlehnung an Max Weber zu begründen, aber bis zum Ende seiner Produktivität um 1930 wußte er sie nie in eine zur Veröffentlichung gereifte Gesamtdarstellung zu gießen. Von Hintze stammen wegweisende Aufsätze zum Verhältnis von Staats- und Heeresverfassung, zur Geschichte der Repräsentation oder zum Wesen des modernen Staates ${ }^{10}$.

8 Grundlegend zu diesem Prozeß: Böckenförde, Die deutsche verfassungsgeschichtliche Forschung (wie Anm. 3).

9 Georg Waitz: Deutsche Verfassungsgeschichte. Die Verfassung des deutschen Volkes in ältester Zeit. 8 Bde., Berlin 1844-1877//31880-1896. Eine neuere wissenschaftliche Monographie über Person und Werk von Georg Waitz fehlt. Zuletzt als Skizze: Jürgen Weitzel: Georg Waitz (1813-86), Deutsche Verfassungsgeschichte. In: Volker Reinhardt (Hg.), Hauptwerke der Geschichtsschreibung, Stuttgart 1997, S. 707-710.

10 Die Grundinformationen zur Person Hintzes liefert: Wolfgang Neugebauer: Hintze, Otto. In: Deutsche Biographische Enzyklopädie 5 (1997), Sp. 56 f. Zuletzt die informative Skizze: ders.: Otto Hintze (1861-1940). In: Michael Fröhlich (Hg.), Das Kaiserreich. 
Hintzes bekanntester Schüler und Berliner Lehrstuhlnachfolger, Fritz Hartung, ging andere Wege. Besonders im Hinblick auf die komparativen Ansätze und die soziologischen Bestandteile bei Hintze handelte es sich um eine methodische und inhaltliche Reduktion $^{11}$. Es war ein Rückzug auf die klassische staatliche Entwicklungsgeschichte mit dem Schwerpunkt auf Staatsform, Staatsorganisation und Staatsverwaltung. Hartungs früher Gesamtüberblick über die »Verfassungsgeschichte vom 15. Jahrhundert bis zur Gegenwart« aus dem Jahre 1914 erlebte mehrere Neuauflagen und blieb bis in die dreißiger Jahre das unumstrittene Standardwerk.

Dies verwundert um so weniger, als man für die zwanziger Jahre generell von einer deutlichen Flaute einer spezifisch verfassungsgeschichtlich ausgerichteten historischen Forschung sprechen muß. Die wenigen einschlägigen Studien waren oftmals positivistisch geraten bzw. sie behandelten Themen nahe dem ideen- oder politikgeschichtlichen Bereich. Die Parteiengeschichte, nicht selten biographisch ausgerichtet, steckte noch in den Anfängen. Strukturanalysen des (Weimarer) Parteiensystems lieferten Außenseiter: Ludwig Bergsträsser, Ernst Fraenkel und Sigmund Neumann waren erste Vertreter einer noch nicht als Lehrfach etablierten Politikwissenschaft wie sie an der »Deutschen Hochschule für Politik« in Berlin betrieben wurde ${ }^{12}$.

Das Jahr 1918 ist somit nicht eigentlich als historiographiegeschichtliche Zäsur zu betrachten. Denn es dominierten die methodischen Traditionen aus dem späten Kaiserreich; neue Denkansätze, u.a. von der Soziologie, wurden weithin scharf zurückgewiesen. Wirkliches Neuerungspotential besaß demgegenüber die juristische Verfassungsgeschichtsschreibung, die von den methodischen Grundlagendebatten des Fachs seit Mitte der zwanziger Jahre inspiriert wurde.

\section{Verfassungsbegriff und Verfassungsgeschichte}

In den 1920er Jahren vollzog sich ein grundlegender gesellschaftlicher und intellektueller Wandel, der sich wissenschaftsgeschichtlich in Abkehr und Neubeginn zugleich auswirkte. Der unter dem Stichwort »Methoden- und Richtungsstreit« in der juristischen Disziplingeschichte geläufige Umschwung war eine wissenschaftliche Kehrtwende in vielfacher Hinsicht ${ }^{13}$. Es vollzog sich die Abwendung vom Historismus in

Portrait einer Epoche in Biographien, Darmstadt 2001, S. 286-298. Eine ausführliche Biographie fehlt bisher. Wichtig vor allem: Wolfgang Neugebauer: Otto Hintze und seine Konzeption der »Allgemeinen Verfassungsgeschichte der neueren Staaten«. In: Zeitschrift für historische Forschung 20 (1993), S. 65-96 [erweitert auch in: Otto Hintze: Allgemeine Verfassungs- und Verwaltungsgeschichte der neueren Staaten. Fragmente. Bd. 1, hg. v. dems./Giuseppe di Costanzo/Michael Erbe, Neapel 1998, S. 35-83].

11 Gerhard Oestreich: Fritz Hartung als Verfassungshistoriker (1883-1967). In: Der Staat 7 (1968), S. 447-469; Werner Schochow: Ein Historiker in der Zeit. Versuch über Fritz Hartung (1883-1967). In: Jahrbuch für die Geschichte Mittel- und Ostdeutschlands 32 (1983), S. 219-250.

12 Vgl. dazu Bleek, Politikwissenschaft (wie Anm. 2), S. 198-228.

13 Die zeitgenössische Verwendung des Begriffs bei Erich Schwinge: Der Methodenstreit in der heutigen Rechtswissenschaft, Bonn 1930. Die differierenden Positionen sind dargestellt bei Michael Stolleis: Geschichte des Öffentlichen Rechts in Deutschland. Bd. 3: 
der Geschichte, vom Neukantianismus in der Philosophie und vom Positivismus in der Rechtswissenschaft. Die als existentiell empfundenen Erfahrungen des Ersten Weltkriegs und seine politisch-gesellschaftlichen Folgen stellten eine Grunderfahrung der Zwischenkriegszeit dar und prägten das wissenschaftliche Denken. Die Weltkriegszäsur hinterließ im Geistesleben tiefe Spuren und wirkte sich forschungsleitend aus. »Die geistige Krisis unserer Zeit«, von der der Historiker Friedrich Meinecke $\operatorname{sprach}^{14}$, war in den publizistischen wie wissenschaftlichen Reden und Veröffentlichungen der Historiker und Juristen in den zwanziger Jahren überall zu greifen.

Man forderte eine »geisteswissenschaftliche $«$ Methode $^{15}$, die Hinwendung der Wissenschaften zum Leben und die Entwicklung einer »Wirklichkeitswissenschaft «, wie sie der »konservative Revolutionär« Hans Freyer für den Aufbau einer »deutschen Soziologie $\ll 1930$ propagierte ${ }^{16}$. Die dominierende positivistische Staatsrechtslehre wurde mit einem soziologisch begründeten Rechtsdenken konfrontiert. Man argumentierte mit Ganzheitsbezug sowie Volks- und Lebensnähe.

Vor diesem Wandel auf dem wissenschaftlichen >Ideenmarkt< vollzog sich die Entstehung eines neuen Verständnisses des Begriffs der Verfassung und in dessen Folge die Etablierung einer neuartigen Verfassungsgeschichtsschreibung. Der prononcierteste Innovator auf dem Feld der Verfassungstheorie war der Staatsrechtler Carl Schmitt ${ }^{17}$. Sein in der Abkehr vom Positivismus entstandener weitgefaßter Verfassungsbegriff war deutlich im Vorfeld juristisch exakt faßbarer Normierung angesiedelt und rekurrierte auf die fundamentale politische Dezision eines Volkes. Schmitt folgerte einen grundlegenden Unterschied zwischen der Verfassung als einer im metajuristischen Bereich getroffenen Grund- und Gesamtentscheidung einerseits und dem Verfassungsgesetz als einem

Staats- und Verwaltungsrechtswissenschaft in Republik und Diktatur 1914-1945, München 1999, S. 158-186. Die Literatur zum Methodenstreit ist insgesamt kaum mehr überschaubar. Zusammenfassend: Max-Emanuel Geis: Der Methoden- und Richtungsstreit in der Weimarer Republik. In: Juristische Schulung 29 (1989), S. 91-96; ausführlicher: Wolfgang März: Der Richtungs- und Methodenstreit der Staatslehre, oder der staatsrechtliche Antipositivismus. In: Knut Wolfgang Nörr/Bertram Schefold/Friedrich Tenbruck (Hg.), Geisteswissenschaften zwischen Kaiserreich und Republik, Stuttgart 1994 (= Methoden der Geisteswissenschaften), S. 75-133.

14 Friedrich Meinecke: Kausalitäten und Werte in der Geschichte. In: Historische Zeitschrift 137 (1928), S. 1-27, hier S. 1, Anm. 1.

15 Die Begriffsprägung stammt von Günther Holstein: Von Aufgaben und Zielen heutiger Staatsrechtswissenschaft. In: Archiv des öffentlichen Rechts 50 (1926), S. 1-40, hier S. 31.

16 Hans Freyer: Soziologie als Wirklichkeitswissenschaft. Logische Grundlegung des Systems der Soziologie, Leipzig 1930/Stuttgart ${ }^{2} 1964$.

17 Seit seinem Tod 1986 ist eine Flut von Sekundärliteratur erschienen. Prägnanter Überblick: Reinhard Mehring: Carl Schmitt zur Einführung, Hamburg 1992/2001; weiterhin: Helmut Quaritsch: Positionen und Begriffe Carl Schmitts, Berlin ${ }^{3} 1995$; Hasso Hofmann: Legitimität gegen Legalität. Der Weg der politischen Philosophie Carl Schmitts, Berlin ${ }^{2} 1992$; Andreas Koenen: Der Fall Carl Schmitt. Sein Aufstieg zum »Kronjuristen des Dritten Reiches «, Darmstadt 1995. Der wichtigste Sammelband: Helmut Quaritsch (Hg.): Complexio Oppositorum. Über Carl Schmitt. Vorträge und Diskussionsbeiträge des 28. Sonderseminars 1986 der Hochschule für Verwaltungswissenschaften Speyer, Berlin 1988 (= Schriftenreihe der Hochschule Speyer, 102). Mit neuen Erkenntnissen: Dirk Blasius: Carl Schmitt. Preußischer Staatsrat in Hitlers Reich, Göttingen 2001. 
daraus resultierenden normlogischen Fixpunkt der Gesetzgebung andererseits. Die Verfassung in Gestalt eines formalen Gesetzes wurde gleichsam ausgespielt gegen die materielle, quasi übergesetzliche Verfassung. Die Verfassung sei, wie Schmitt formulierte, der »konkrete Gesamtzustand politischer Einheit und sozialer Ordnung«. Verfassungsrecht und Verfassungswirklichkeit traten in der Konsequenz auseinander. Eine Verfassungsgeschichtsschreibung, die sich diesem positiven Verfassungsbegriff verpflichtet wußte, konnte sich mit der Totalität der menschlichen Existenz beschäftigen, mußte über eine rein politisch-rechtlich fundierte Institutionengeschichte hinausgehen und zu einer »histoire constitutionnelle totale« werden.

Nahezu zeitgleich mit Schmitts »Verfassungslehre« erschien Rudolf Smends Monographie über »Verfassung und Verfassungsrecht«. Der Göttinger Staatsrechtler verstand die Verfassung als lebendigen Prozeß einer stets wiederholten Integration verschiedener politischer Lebensäußerungen in Staat und Gesellschaft. Smends Verfassungslehre kritisierte die Weimarer Reichsverfassung wegen ihres Mangels an organischem Gehalt. Statt eines juristischen schwebte Smend ein soziologisch definierter Verfassungsbegriff vor. Anklänge an ständestaatliche Konzepte oder faschistische Vorbilder sind dabei nicht von der Hand zu weisen ${ }^{18}$.

Fritz Hartung zählte zu den an der Verfassungsmaterie sogleich interessierten Historikern. Seine Reaktion auf die verfassungstheoretischen Neuentwürfe von Schmitt und Smend wurde in einem führenden Fachorgan, der »Zeitschrift für die gesamte Staatswissenschaft «, veröffentlicht und ließ an Deutlichkeit wenig zu wünschen übrig ${ }^{19}$. Die beiden Monographien seien »Ausdruck des lebendigen Ringens um eine neue Grundlegung der Staatslehre [und] Zeichen der Besinnung auf das Wesen des Staates«. Insgesamt reagierte Hartung abwartend-kritisch: so konstatierte er, daß die von Schmitt betonte Unterscheidung zwischen Verfassung und Verfassungsgesetz »freilich nicht eben neu« sei. Im Kern sei Schmitts Verfassungslehre jedenfalls »nicht als wissenschaftlich fruchtbar« einzustufen. Auch Otto Hintzes Besprechung von Schmitt in der »Historischen Zeitschrift« war bei aller Anerkennung nicht wirklich positiv ${ }^{20}$. Wie Sensoren reagierten mit Hintze und Hartung gerade die beiden führenden deutschen

18 Rudolf Smend: Verfassung und Verfassungsrecht. In: ders.: Staatsrechtliche Abhandlungen und andere Aufsätze, Berlin ${ }^{2}$ 1968, S. 119-276 [zuerst 1928]. Zu Person und Werk: E.[rnst] R.[udolf] Huber: Rudolf Smend. In: Jahrbuch der Akademie der Wissenschaften in Göttingen für das Jahr 1976, Göttingen 1977, S. 105-121; Peter Badura: Staat, Recht und Verfassung in der Integrationslehre. Zum Tode von Rudolf Smend (15. Januar $1882-5$. Juli 1975). In: Der Staat 16 (1977), S. 305-325; Manfred Friedrich: Rudolf Smend. In: Archiv des öffentlichen Rechts 112 (1987), S. 1-25.

19 Fritz Hartung: Verfassungslehre [Rez. zu Carl Schmitt, Verfassungslehre]. In: Zeitschrift für die gesamte Staatswissenschaft 87 (1929), S. 225-239. Einschlägig für die Rezeption der Verfassungslehre Carl Schmitts durch Hartung und Hintze: Hans-Christof Kraus: Verfassungslehre und Verfassungsgeschichte. Otto Hintze und Fritz Hartung als Kritiker Carl Schmitts. In: Dietrich Murswiek/Ulrich Storost/Heinrich A. Wolff (Hg.), Staat - Souveränität - Verfassung. Festschrift für Helmut Quaritsch zum 70. Geburtstag, Berlin 2000 (= Schriften zum Öffentlichen Recht, 814), S. 637-661. Ich weiche in der Einschätzung von Kraus' Urteil ab.

20 Otto Hintze: Rez. zu Carl Schmitt, Verfassungslehre. In: Historische Zeitschrift 139 (1929), S. 562-568. 
Verfassungshistoriker auf die grundlegenden Begriffsdebatten im Nachbarfach. Derartige Neudefinitionsversuche und die auf ihnen aufbauenden Neuentwürfe der juristischen Verfassungshistoriographie ließen spätestens seit Mitte der 1930er Jahre die historische Traditionslinie der Verfassungsgeschichtsschreibung deutlich in die Defensive geraten.

\section{Verfassungsgeschichte als Grundlagenfach im Nationalsozialismus}

Der Durchbruch und die nachfolgende Dominanz einer von Juristen betriebenen Verfassungsgeschichte erfolgte nicht zufällig nach der Machtübernahme der Nationalsozialisten im Jahr 1933. Carl Schmitt avancierte zum staatsrechtlichen Kronjuristen des Regimes $^{21}$. Auf dem Gebiet der Verfassungsgeschichte hat sich Schmitt indes auffälligerweise fast ganz zurückgehalten. Allein seine kurze Monographie über »Staatsgefüge und Zusammenbruch des zweiten Reiches « gibt eine Deutung des Konstitutionalismus als staatsrechtlicher Durchgangsstation zwischen Absolutismus und Demokratie, die bereits unter Zeitgenossen nicht wenig umstritten war (und im übrigen bis heute eine kontrovers diskutierte Position der Verfassungsgeschichte markiert) ${ }^{22}$. Fritz Hartung veröffentlichte in der »Historischen Zeitschrift« eine geharnischte Kritik. Schmitt weiche mit seiner voreingenommenen Quellenauswertung von den »Grundvoraussetzungen unserer wissenschaftlichen Arbeit« ab. Er benutze zudem Begriffe »aus der politischen Terminologie unserer Tage«. Eine »ihrer Verantwortung bewußte Wissenschaft « müsse dagegen »Einspruch« erheben, da sonst eine »Gefahr für die politische Bildung der heranwachsenden Generation $«$ drohe $^{23}$.

Doch im preußischen Unterrichtsministerium stieß derartige fachwissenschaftliche Kritik auf taube Ohren. Im Gegenteil: Die wissenschaftspolitischen Rahmenbedingungen förderten die intellektuelle Wirkmächtigkeit des Verfassungsbegriffs Schmittscher Provenienz. Im Gefolge des mächtigen Staatsrechtlers gelang es einem Schülerkreis jüngerer Wissenschaftler, zumeist ehemaligen Anhängern der sogenannten Konservativen Revolution, bereits in den Anfangsjahren Lehrstühle entlassener Hochschullehrer

21 Koenen (wie Anm. 17); Blasius (wie Anm. 17).

22 Carl Schmitt: Staatsgefüge und Zusammenbruch des zweiten Reiches. Der Sieg des Bürgers über den Soldaten, Hamburg 1934 (= Der deutsche Staat der Gegenwart, 6). Die kontroversen Positionen zuerst bei Ernst Rudolf Huber: Deutsche Verfassungsgeschichte seit 1789. Bd. 3: Bismarck und das Reich, Stuttgart usw. 1963, S. VII f., 3-26; Ernst-Wolfgang Böckenförde: Der Verfassungstyp der deutschen konstitutionellen Monarchie im 19. Jahrhundert. In: ders. (Hg.), Moderne deutsche Verfassungsgeschichte (1815-1914), Königstein/Ts. ${ }^{2} 1981$ (= Neue wissenschaftliche Bibliothek, 51), S. 146-170 [zuerst 1967]. Bei Elisabeth Fehrenbach: Verfassungsstaat und Nationsbildung, München 1992 (= Enzyklopädie deutscher Geschichte, 22), S. 71-85, erfolgt eine perspektivenreiche wissenschaftsgeschichtliche Einbettung der Kontroverse.

23 Fritz Hartung: Staatsgefüge und Zusammenbruch des zweiten Reiches. In: Historische Zeitschrift 151 (1935), S. 528-544, hier S. 543 f. Ausführliche Einordnung der Rezension bei Hans-Christof Kraus: Soldatenstaat oder Verfassungsstaat? Zur Kontroverse zwischen Carl Schmitt und Fritz Hartung über den preußisch-deutschen Konstitutionalismus (1934/ 35). In: Jahrbuch für die Geschichte Mittel- und Ostdeutschlands 45 (1999), S. 275-310. 
zu übernehmen. In den ersten Jahren des neuen Regimes kam es bei der Neubesetzung der Katheder des öffentlichen Rechts geradezu zu einem Wettbewerb »um die wenigen bekannten und eindeutig nat.[ional]-soz.[ialistischen] Juristen $\ll^{24}$. Ernst Rudolf Huber und Ernst Forsthoff erhielten bereits mit dreißig Jahren 1933 die Lehrstühle der demokratischen Professoren Walther Schücking in Kiel und Hermann Heller in Frankfurt.

Begleitet wurde der Generationswechsel im Sinne des nationalsozialistischen Regimes von einer wissenschaftspolitischen Umgestaltung, von der im Bereich der Rechtswissenschaft vor allem die historischen Disziplinen profitierten. Dies war ganz offensichtlich dem neuen >starken Mann< im Reichsministerium für Erziehung und Wissenschaft, dem Rechtshistoriker Karl August Eckhardt, zu verdanken. Seine wissenschaftspolitischen Leitlinien wirkten sich personell wie institutionell maßgeblich auf die Etablierung der Verfassungsgeschichte als Lehrfach aus. Denn zum einen war er als Hauptreferent im Amt Wissenschaft für die Besetzung der Lehrstühle sowohl in den Rechts- wie in den Geschichtswissenschaften zuständig. Zum anderen war er der Spiritus rector der juristischen Studienreform des Jahres $1935^{25}$.

Auf einer Tagung der Reichsfachgruppe Hochschullehrer des Bundes Nationalsozialistischer Deutscher Juristen vom Dezember 1934 wurde über die Einführung einer neuen Studienordnung auf Reichsebene diskutiert. Unter Leitung Carl Schmitts verstand sich die Reichsfachgruppe als »nationalsozialistische Kampfzelle«, die sich der »Neugestaltung des juristischen Bildungswesens [...] in besonderem Maße annehmen « wolle ${ }^{26}$. Das Ergebnis der Beratung waren die »Richtlinien für das Studium der Rechtswissenschaft«, die Reichsminister Bernhard Rust am 18. Januar 1935 verkündete. In ihnen wurde die Rechtswissenschaft in ihren Grundfesten historisiert, d.h. es wurden Vorgeschichte, Antike und Germanische Rechtsgeschichte, Privatrechtsgeschichte der Neuzeit, Neueste politische Geschichte und die Verfassungsgeschichte der Neuzeit als Grundlagenfächer in den Vorlesungskanon vornehmlich der frühen juristischen Fachsemester festgeschrieben. Jeder Student solle »die völkischen« - gemeint waren damit besonders auch die historischen - »Grundlagen der Wissenschaft kennen lernen. « ${ }^{27}$

24 Sächsisches Hauptstaatsarchiv Dresden, Best. Ministerium für Volksbildung, Nr. 10.200/ 48, fol. 11-17 (30.11.1934), im Zusammenhang mit der Kandidatenliste für das öffentlichrechtliche Ordinariat in Leipzig.

25 Biographisch ausführlich: Hermann Nehlsen: Karl August Eckhardt †. In: Zeitschrift der Savigny-Stiftung für deutsche Rechtsgeschichte/Germanistische Abteilung 104 (1987), S. 497-536. Dazu ergänzend: Hermann Krause: Karl August Eckhardt. In: Deutsches Archiv für Erforschung des Mittelalters 35 (1979), S. 1-16; zuletzt: Anna-Maria Gräfin von Lösch: Der nackte Geist. Die Juristische Fakultät der Berliner Universität im Umbruch von 1933, Tübingen 1999 (= Beiträge zur Rechtsgeschichte des 20. Jahrhunderts, 26), S. 405-426, 440-447.

26 Carl Schmitt: Bericht über die Fachgruppe Hochschullehrer im BNSDJ. In: Deutsches Recht 4 (1934), S. 17.

27 Karl August Eckhardt: Das Studium der Rechtswissenschaft, Hamburg 1935 (= Der deutsche Staat der Gegenwart, 11), S. 9. Zu den Studienordnungen: Ralf Frassek: Weltanschaulich begründete Reformbestrebungen für das juristische Studium in den 30er und 40er Jahren. In: Zeitschrift der Savigny-Stiftung für deutsche Rechtsgeschichte/Germanistische Abteilung 111 (1994), S. 564-591; ders.: Steter Tropfen höhlt den Stein - Juristenausbildung im Nationalsozialismus und danach. In: ebd. 117 (2000), S. 294-355; ders.: Juristenausbildung im Nationalsozialismus. In: Kritische Justiz 37 (2004), S. 85-96. 
Die kämpferisch-programmatische Popularisierung der neuen Studienordnung erfolgte in zwei Heften der weit verbreiteten Broschürenreihe »Der deutsche Staat der Gegenwart«. Diese Reihe wurde von Carl Schmitt herausgegeben und erschien im Hausverlag mehrerer juristischer Partei- und Staatsstellen, der Hanseatischen Verlagsanstalt. Mit dem Aufruf »Die deutsche Rechtswissenschaft muß nationalsozialistisch werden«, wurden die Richtlinien nebst einem Studienplan eingeleitet ${ }^{28}$. Karl August Eckhardt ließ sein Referat auf der Tagung vom Dezember 1934 mitabdrucken. Zur historischen Erweiterung des Rechtsstudiums hieß es darin: »Ferner muß jeder Student nach meiner Ansicht eine historische Vorlesung, möglichst über die Geschichte des neunzehnten Jahrhunderts, hören. Gerade auf historischem Gebiete ist das Unwissen der Studenten am katastrophalsten. Viele wissen z.B. nicht, was der Deutsche Bund ist. Ich habe die tollsten Dinge auf diesem Gebiete erlebt und jeder von Ihnen kann mir diese Wahrnehmung bestätigen. $\ll^{29}$

Nur ein Jahr später äußerte sich auch Carl Schmitt als Reichsfachgruppenleiter in einem programmatischen Beitrag »über die neuen Aufgaben der Verfassungsgeschichte «. »Das neue Fach« solle die bisher übliche »Trennung einer 'rein juristischen' von einer 'rein geschichtlichen' Betrachtungsweise« überwinden. Es müsse ein »zusammenfassendes Geschichtsbild entstehen, das die Rechtsentwicklung als eine Schöpfung deutschen Lebens in ihrer volklichen Einheit erkennen läßt«. Dabei solle die Darstellung weit über das hinausgreifen, was man bisher unter dem »'konstitutionalistischen' Verfassungsbegriff « subsumiert habe. »In der neuen Verfassungsgeschichte wird sich der nationalsozialistische, nicht mehr liberale, auch nicht mehr nationalliberale, und nicht mehr freimaurerisch-demokratische Verfassungsbegriff rechtswissenschaftlich bewähren müssen, indem er sich auf die Einheit und Ganzheit der Lebensordnung des deutschen Volkes richtet.« Es stehe ein »Umdenken und Umpflügen der überlieferten Begriffe« bevor. »Mit dem Lehrfach 'Verfassungsgeschichte' beginnt hoffentlich auch ein neuer Abschnitt der Geschichtswissenschaft. « ${ }^{30}$

Die Folge der Studienplanreform war die Entstehung neuer Überblicksdarstellungen zur Verfassungsgeschichte. Schließlich hatte die Reichsfachgruppe Hochschullehrer ganz besonders eine »Reform der Lehrmittel, insbesondere der Lehrbücher « im Auge gehabt $^{31}$. Der Grundriß des Königsberger Staatsrechtlers Ernst Forsthoff aus dem Jahr 1940 bildet das repräsentativste Beispiel der Zeit ${ }^{32}$. Forsthoffs Darstellung war ganz aus der entsprechenden Vorlesung des zweiten juristischen Semesters entstanden. Sie endete mit Bismarcks Reichsgründung, weil das Verfassungsrecht des Kaiserreichs in der Vorlesung über »Verfassung « behandelt werden sollte. Angestrebt war ein Durch-

28 Eckhardt (wie Anm. 27), S. 9.

29 Ebd., S. $30 \mathrm{f}$.

30 Carl Schmitt: Über die neuen Aufgaben der Verfassungsgeschichte. In: ders., Positionen und Begriffe im Kampf mit Weimar - Genf - Versailles 1923-1939, Hamburg 1940, Ndr. Berlin 1988, S. 229-234 [zuerst in: Jahrbuch der Akademie für Deutsches Recht 3 (1936), S. 10-15].

31 Schmitt, Bericht über die Fachgruppe (wie Anm. 26).

32 Ernst Forsthoff: Deutsche Verfassungsgeschichte der Neuzeit, Berlin 1940. Die vierte Auflage (Stuttgart usw. 1972) ist bis heute lieferbar. 
gang durch die deutsche Geschichte der Neuzeit unter besonderer Berücksichtigung der politischen, sozialen und geistigen Verfassung der jeweiligen Epoche. Leitbegriff war, ganz dem Credo der sogenannten ordotheoretischen Richtung der Staatsrechtslehre entsprechend, die Ordnung von Volk und Staat, welche sich als verfassungshistorische Kernbestandteile in einem permanenten Kampf, einem Ringen um Verfassung befanden. Bestimmend für die Deutung der deutschen Verfassungsgeschichte war ein selten unterbrochener Dekompositionsprozeß: von »Niedergang «, »Auflösung«, »Zersplitterung «, »Aushöhlung «, »Verfall«, »Krise«, »Scheitern«, »Ende« und »Zusammenbruch« ist allein das Inhaltsverzeichnis übervoll.

Auch Ernst Rudolf Huber, gleichfalls Schüler Carl Schmitts, wandte sich seit Mitte der dreißiger Jahre der Verfassungsgeschichte zu. Anders als Forsthoff, der sich auf das Verwaltungsrecht spezialisierte, hatte sich Huber, ausgehend von einem Experten für das Staatskirchen- und das Wirtschaftsverwaltungsrecht in den späten Weimarer Jahren, im institutionellen Rahmen der sogenannten Kieler »Stoßtruppfakultät« als einer der führenden Vertreter des Verfassungsrechts im »Dritten Reich « exponiert und etabliert $^{33}$. 1937 wechselte Huber an die renommierte juristische Fakultät nach Leipzig und von dort als »einer der bestqualifizierten jüngeren Staatsrechtslehrer« im Jahre 1941 an die »Grenzlanduniversität « Straßburg, wo er bis zur alliierten Besetzung der Stadt Ende 1944 wirkte $^{34}$.

Huber hatte bereits 1932 den Dezionismus Carl Schmitts als zu eng gefaßt kritisiert und auch dessen Verfassungsbegriff lediglich modifiziert übernommen ${ }^{35}$. In seiner eigenen Verfassungstheorie kam eine vom Neuhegelianismus geprägte stärkere Einbeziehung des Staates zum Tragen. 1937 verfaßte er den maßgeblichen Kommentar zum Staatsrecht des Nationalsozialismus, der in zweiter Auflage 1939 anläßlich des Anschlusses von Österreich aktualisiert und erweitert unter dem geänderten Titel als »Verfassungsrecht des Großdeutschen Reiches« $\operatorname{erschien}^{36}$. Das zeitgenössische Urteil

33 Zu Person und Werk: Ralf Walkenhaus: Konservatives Staatsdenken. Eine wissenssoziologische Studie zu Ernst Rudolf Huber, Berlin 1997; Zum Komplex der ,Kieler Schule': Jörn Eckert: Was war die Kieler Schule? In: Franz Jürgen Säcker (Hg.), Recht und Rechtslehre im Nationalsozialismus. Ringvorlesung der Rechtswissenschaftlichen Fakultät der Christian-Albrechts-Universität zu Kiel, Baden-Baden 1992 (= Kieler Rechtswissenschaftliche Abhandlungen, NF 1), S. 37-70; Ralf Walkenhaus: Gab es eine »Kieler Schule«? Die Kieler Grenzlanduniversität und das Konzept der »politischen Wissenschaften« im Dritten Reich. In: Wilhelm Bleek/Hans J. Lietzmann (Hg.), Schulen der deutschen Politikwissenschaft, Opladen 1999, S. 159-182.

34 Generallandesarchiv Karlsruhe, Abt. 235, Nr. 29851 (Heidelberger Dekan Engisch, 24.2.1936). Zu Straßburg: Herwig Schäfer: Juristische Lehre und Forschung an der Reichsuniversität Straßburg 1941-1944, Tübingen 1999 (= Beiträge zur Rechtsgeschichte des 20. Jahrhunderts, 23).

35 Ernst Rudolf Huber: Verfassung und Verfassungswirklichkeit bei Carl Schmitt. In: Blätter für Deutsche Philosophie 5 (1931/32), S. 302-315. Vgl. auch die spätere Kritik: ders.: »Positionen und Begriffe«. Eine Auseinandersetzung mit Carl Schmitt. In: Zeitschrift für die gesamte Staatswissenschaft 101 (1941), S. 1-44.

36 Ernst Rudolf Huber: Verfassung, Hamburg 1937 (= Grundzüge der Rechts- und Wirtschaftswissenschaft, A) [erweitert u.d.T.: Verfassungsrecht des Großdeutschen Reiches, Hamburg $\left.{ }^{2} 1939\right]$. 
des Reichsarbeitsblatts lautete: »Besonders wertvoll sind die den einzelnen Abschnitten vorangeschickten kurzen verfassungsgeschichtlichen Rückblicke « ${ }^{37}$.

Schon 1935 hatte sich der Kieler Staatsrechtler zur Bedeutung des neuen Grundlagenfachs Verfassungsgeschichte geäußert. In der von ihm mitherausgegebenen »Zeitschrift für die gesamte Staatswissenschaft « veröffentlichte Huber einen programmatischen Aufsatz über »die deutsche Staatswissenschaft $\ll^{38}$. Diese sei eine »Wissenschaft von der politischen Totalität des Staates«, eine »politische Wirklichkeitswissenschaft«, deren Grundbegriff das Volk sei. Zur neuen Staatslehre gehöre »als erstes Stück« die Verfassungsgeschichte. Es gehe darum, »in der geschichtlichen Überlieferung die lebendigen und kräftigen Ströme des Volkes, aber auch die in der völkischen Eigenart gegebenen besonderen Gefahren und Zersetzungsmöglichkeiten zu erfassen.« Die Verfassungsgeschichte sei, indem sie »die Kräfte und die Formen des vergangenen Seins erforscht, [...] für eine gegenwartsnahe Staatswissenschaft unentbehrlich.« Gegenwart und Geschichte wurden aufeinander bezogen. Die Verfassungsgeschichte figurierte als die historische Komponente der Staatswissenschaft in ihrer Funktion als integrativer ,Dachwissenschaft ${ }^{39}$. 1937 bei Annahme des Rufs nach Leipzig bat Huber bei der Festlegung des mit der Professur verbundenen Lehrauftrags darum, die Verfassungsgeschichte ausdrücklich einzubeziehen ${ }^{40}$.

Seit etwa 1935 beschäftigte sich Huber intensiv mit der deutschen Militärgeschichte oder, um es in der zeitgenössischen Diktion zu sagen, mit der Geschichte der »Wehrverfassung «. 1938 veröffentlichte er eine rund 450seitige Monographie, die 1943 in zweiter Auflage erschien. Das Werk »Heer und Staat in der deutschen Geschichte « bietet einen militärverfassungsgeschichtlichen Durchgang durch die deutsche Geschichte von den Germanen bis zur Gegenwart, d.h. bis zum Ende des Ersten Weltkriegs ${ }^{41}$. Mit der Verknüpfung von militärischer und politischer Geschichte beschritt Huber indes keinen völlig neuen Weg. So hatte beispielsweise bereits Otto Hintze 1906 die Kohärenz zwischen »Staatsverfassung und Heeresverfassung « dargelegt ${ }^{42}$. Und Carl Schmitt benannte 1934 den Zwiespalt zwischen politischer und militärischer Verfassung als Ursache für »den Zusammenbruch des Zweiten Reiches « ${ }^{43}$. Huber knüpfte an diese Überlegungen an, indem er die Wehrverfassung als »die stärkste unter den verfassungsbildenden Kräften « bezeichnete. Er wolle, so kündigte er an, »nicht nur ein wesentliches Kapitel deutscher Verfassungsgeschichte darstellen, sondern zugleich die Fruchtbarkeit eines Rechtsdenkens erproben, das nicht die abstrakte Norm, sondern die

37 Georg Hartrodt: Rez. zu Ernst Rudolf Huber, Verfassung. In: Reichsarbeitsblatt II (Nichtamtlicher Teil), Jg. 1937, S. 129.

38 Ernst Rudolf Huber: Die deutsche Staatswissenschaft. In: Zeitschrift für die gesamte Staatswissenschaft 95 (1935), S. 1-65.

39 Ebd., S. 1, 28, 50.

40 Tula Huber-Simons in einem Brief an den Verfasser vom 1.4.1997.

41 Ernst Rudolf Huber: Heer und Staat in der deutschen Geschichte, Hamburg 1938/ ${ }^{2} 1943$.

42 Otto Hintze: Staatsverfassung und Heeresverfassung. In: ders., Staat und Verfassung. Gesammelte Abhandlungen zur allgemeinen Verfassungsgeschichte, hg. v. Gerhard Oestreich, mit einer Einleitung v. Fritz Hartung, Göttingen ${ }^{3} 1970$ (= Gesammelte Abhandlungen, 1), S. 52-83 [zuerst 1906].

43 Schmitt, Staatsgefüge (wie Anm. 22). 
lebendige Ordnung als das wirkliche Recht zu begreifen strebt. ${ }^{44} \mathrm{Daß}$ sich Huber Mitte der dreißiger Jahre ausgerechnet zu einem militärgeschichtlichen Thema entschloß, dürfte, so steht zu vermuten, dem Zeitgeist geschuldet sein.

In seinen ideengeschichtlichen Aufsätzen, die vor allem Anfang der vierziger Jahre entstanden, befaßt sich Huber mit dem 17. bis 19. Jahrhundert: Leitthema ist stets das Volk und der Volksgedanke. Es geht in diesen geistesgeschichtlichen Untersuchungen um den Volksbegriff der Reichsrechtswissenschaft des 17. und 18. Jahrhunderts, um den preußischen Staatspatriotismus, die Entstehung des »historisch-individuellen Volksbegriffs « und den »Volksgedanken in der Revolution von $1848 «^{45}$. Huber rezipierte und propagierte damit das Paradigma »Volk«, welches die Geschichtsforschung seit 1933 dominant beherrschte ${ }^{46}$.

»Politische Volksgeschichte heißt das Gebot der Stunde«, formulierte auch der Wiener Mediävist Otto Brunner sein Credo 1939. Der 1941 in Wien zum Ordinarius ernannte frühere Archivar hatte 1939 mit der Publikation von »Land und Herrschaft. Grundfragen der territorialen Verfassungsgeschichte Südostdeutschlands im Mittelalter « den wissenschaftlichen Durchbruch geschafft ${ }^{47}$. Sein methodisches Programm legte er in seinem Aufsatz »Moderner Verfassungsbegriff und mittelalterliche Verfassungsgeschichte « vor ${ }^{48}$. Den Spuren Schmitts und Hubers folgend, versuchte er eine »Verknüpfung von politischem Denken und Geschichtsauffassung «. Seiner Forderung

44 Huber, Heer (wie Anm. 41), S. 8, 11.

45 Ernst Rudolf Huber: Der Volksgedanke in der Revolution von 1848. In: Zeitschrift für die gesamte Staatswissenschaft 99 (1938/39), S. 393-439 [gekürzt auch in: Paul Ritterbusch (Hg.), Politische Wissenschaft, Berlin/Leipzig/Wien 1940, S. 9-26]; ders.: Reich, Volk und Staat in der Reichsrechtswissenschaft des 17. und 18. Jahrhunderts. In: Zeitschrift für die gesamte Staatswissenschaft 102 (1942), S. 593-627; ders.: Aufstieg und Entfaltung des deutschen Volksbewußtseins, Straßburg 1942 (= Straßburger Universitätsreden, 2); ders.: Der preußische Staatspatriotismus im Zeitalter Friedrichs des Großen. In: ebd. 103 (1943), S. 430-468; ders.: Lessing, Klopstock, Möser und die Wendung vom aufgeklärten zum historisch-individuellen Volksbegriff. In: ebd. 104 (1944), S. 121-159.

46 Willi Oberkrome: Volksgeschichte. Methodische Innovation und völkische Ideologisierung in der deutschen Geschichtswissenschaft 1918-1945, Göttingen 1993 (= Kritische Studien zur Geschichtswissenschaft, 101); Ingo Haar: Historiker im Nationalsozialismus. Deutsche Geschichtswissenschaft und der »Volkstumskampf « im Osten, Göttingen 2000 (= Kritische Studien zur Geschichtswissenschaft, 143).

47 Otto Brunner: Land und Herrschaft. Grundfragen der territorialen Verfassungsgeschichte Südostdeutschlands im Mittelalter, Wien 1939 (=Veröffentlichungen des österreichischen Instituts für Geschichtsforschung, 1), S. 157. Zu Person und Werk: Reinhard Blänkner: Von der »Staatsbildung « zur »Volkwerdung«. Otto Brunners Perspektivenwechsel der Verfassungshistorie im Spannungsfeld zwischen völkischem und alteuropäischem Geschichtsdenken. In: Luise Schorn-Schütte (Hg.), Alteuropa oder Frühe Moderne. Deutungsmuster für das 16. bis 18. Jahrhundert aus dem Krisenbewußtsein der Weimarer Republik in Theologie, Rechts- und Geschichtswissenschaft, Berlin 1999 (= Zeitschrift für historische Forschung; Beih., 23), S. 87-135; Hans Boldt: Otto Brunner und die deutsche Verfassungsgeschichte. In: Karl-Egon Lönne (Hg.), Historismus in den Kulturwissenschaften, Tübingen/Basel 2003, S. 193-206.

48 Otto Brunner: Moderner Verfassungsbegriff und mittelalterliche Verfassungsgeschichte. In: Mitteilungen des Instituts für österreichische Geschichtsforschung, Erg.-Bd. 14 (1939), S. 513-528. 
nach einer möglichst quellennahen Begriffssprache wird noch heute bahnbrechende Bedeutung zugemessen. Zeitgenössisch war damit explizit auch die Beseitigung der liberal geprägten juristischen Terminologie des 19. Jahrhunderts gemeint. Der Historie werde somit »der Weg zu einem neuen Verständnis der Vergangenheit und zugleich zum Dienst an der Gegenwart eröffnet $~{ }^{49}$. Der Altmeister der deutschen Rechtsgeschichte, Heinrich Mitteis, würdigte in einer ansonsten kritischen Besprechung Brunners Buch, weil es »zu einem völligen Neubau der deutschen Verfassungsgeschichte« beitragen könne. Es sei das erste Werk, »das für die verfassungsgeschichtliche Forschung die Staatsrechtslehre des neuen Deutschland fruchtbar macht $~^{50}$. Mit dem Kriegsende und dem Zusammenbruch des Deutsches Reiches 1945 wurden Überlegungen für eine weitere politische Instrumentalisierung der Verfassungsgeschichte jedoch obsolet.

\section{Die >Rückkehr< der Verfassungsgeschichte nach 1945}

Wie generell stellt das Jahr 1945 auch für die Wissenschaftsgeschichte nur sehr bedingt einen Neubeginn und eine Zäsur dar. Nicht wenige wissenschaftliche Traditionen und Personen vermochten zu überleben. Dies gelang denjenigen konservativer oder nationaler Provenienz und Geisteshaltung zumal dann, wenn sie sich rückblickend in eine partielle Opposition zum Regime zu stellen vermochten.

In inhaltlich-methodischer Hinsicht dominierten nach 1945 in der Geschichtswissenschaft zunächst die Forschungen in der Tradition des Historismus mit den Schwerpunkten auf politik- bzw. ideengeschichtlichem Feld ${ }^{51}$. Die Verfassungsgeschichte von Wissenschaftlern wie Huber oder Brunner war als reine Formgeschichte diskreditiert und zudem kontaminiert durch die politische Einstellung ihrer Protagonisten vor 1945. Verfassungsgeschichte war nun vornehmlich als »Demokratie-Geschichte« möglich. Untersuchungen über die freiheitlich-liberalen Traditionen der deutschen Geschichte und Studien zu den Ursachen des Untergangs der ersten deutschen Demokratie standen im Vordergrund der Forschungsbemühungen. Institutionell kam solchen Unternehmungen vor allem die Gründung der »Kommission für Geschichte des Parlamentarismus und der politischen Parteien « in Bonn im Jahre 1951 zugute $^{52}$.

Nicht wenige Fachvertreter, vor allem der Schülergeneration von Fritz Hartung, hatten sich derweil in das Arkanum der Frühen Neuzeit oder der Wirtschaftsgeschichte geflüchtet ${ }^{53}$. Als verfassungshistorische Studien auch über das 19. und 20. Jahrhundert seit Beginn der sechziger Jahre wieder etwas mehr Konjunktur hatten, standen sie,

49 Ebd., S. 528.

50 Heinrich Mitteis: Land und Herrschaft. Bemerkungen zu dem gleichnamigen Buch Otto Brunners. In: Historische Zeitschrift 163 (1941), S. 255-281, 471-489, hier S. 256 f.

51 Winfried Schulze: Deutsche Geschichtswissenschaft nach 1945, München 1989 (= Historische Zeitschrift, Beih. 10).

52 Martin Schumacher: Gründung und Gründer der Kommission für Geschichte des Parlamentarismus und der politischen Parteien. In: Karl Dietrich Bracher u.a. (Hg.), Staat und Parteien. Festschrift für Rudolf Morsey zum 65. Geburtstag, Berlin 1992, S. 1029-1054.

53 Zum Beispiel Carl Hinrichs oder Wilhelm Treue. 
zumal aus Sicht von Politikwissenschaftlern und Sozialhistorikern, im Verdacht konservativer Rechtfertigungsstrategien.

In personeller Hinsicht hatten nur wenige Vertreter ihres Faches nach einigen Jahren noch unter den Folgen ihrer exponierten Position vor 1945 zu leiden. Fast alle saßen 1950 bereits wieder auf Lehrstühlen und befanden sich auch wissenschaftspolitisch schnell wieder an den Schlüsselstellen. Nicht ganz so umstandslos gelang den maßgeblichen Verfassungshistorikern der NS-Zeit die Rückkehr in die universitäre Wissenschaft. Ernst Forsthoff mußte bis 1953 warten, ehe er in Heidelberg einen Lehrstuhl erhielt ${ }^{54}$, Otto Brunner kam erst 1954 nach Hamburg. Am beschwerlichsten war der Weg für Ernst Rudolf Huber. Ihm gelang es zunächst nur, seit 1952 in Freiburg einen Lehrauftrag für Verfassungsgeschichte zu erhalten. Nach weiteren fünf Jahren wurde er schließlich - und auch dies nicht ohne Probleme - auf eine Professur an die winzige Hochschule für Sozialwissenschaften in Wilhelmshaven berufen. Seine Rückkehr an eine etablierte juristische Fakultät erfolgte mit der Eingliederung Wilhelmshavens in die Göttinger Universität $1962^{55}$. Es steht zu vermuten und ist in Einzelfällen auch nachweisbar, daß netzwerkartige Strukturen aus der Zeit vor 1945 die Rückkehr der kurzzeitig wissenschaftlich Verbannten nach 1950 begünstigten, die »Restauration « ermöglichten oder gar forcierten.

Forsthoff, Brunner und Huber, die »alten Kämpfer« der Verfassungsgeschichtsschreibung im Nationalsozialismus, vermochten vor allem seit Ende der fünfziger Jahre weitreichende Wirkungen in ihren Forschungsgebieten zu entfalten: Forsthoff auf dem Gebiet des Verwaltungsrechts, Brunner im Bereich der Sozialgeschichte, Huber in der Verfassungsgeschichte. Ernst Rudolf Hubers Ruhm verbindet sich bis heute mit einem Mammutwerk, das er Anfang der fünfziger Jahre im Alleingang in Angriff nahm und in insgesamt sieben Textbänden von über 7.700 Seiten Umfang erst 1984 abschloß: der »Deutschen Verfassungsgeschichte seit 1789 «, die er bis ins Jahr 1933 bearbeitete. Inhaltlich wie methodisch knüpfte er dabei an seine Untersuchungen aus den dreißiger Jahren an, dehnte aber die Darstellung auf eine umfassende Studie zur politischen, Sozial- und Wirtschafts-, Militär- und Bildungsverfassung inklusive Ideengeschichte von nahezu eineinhalb Jahrhunderten aus. Im Zentrum stand die als geschlossenes System interpretierte Staatsform des deutschen Konstitutionalismus der zweiten Hälfte des 19. Jahrhunderts ${ }^{56}$. Erst mit der Gegenposition von Ernst-Wolfgang

$54 \mathrm{Zu}$ den Bemühungen, ihn in Frankfurt zu etablieren: Notker Hammerstein: Die Johann Wolfgang Goethe-Universität Frankfurt am Main. Von der Stiftungsuniversität zur staatlichen Hochschule. Bd. 1: 1914 bis 1950, Neuwied/Frankfurt a.M. 1989, S. 777 ff.

55 Ewald Grothe: Eine ,lautlose' Angelegenheit? Die Rückkehr des Verfassungshistorikers Ernst Rudolf Huber in die universitäre Wissenschaft nach 1945. In: Zeitschrift für Geschichtswissenschaft 47 (1999), S. 980-1001.

56 Hartwig Brandt: Ernst Rudolf Hubers »Deutsche Verfassungsgeschichte«. Eine methodologische Betrachtung. In: Vierteljahrsschrift für Sozial- und Wirtschaftsgeschichte 74 (1987), S. 229-241; Hans Boldt: Rez. zu Ernst Rudolf Huber, Deutsche Verfassungsgeschichte seit 1789, 7 Bde., Stuttgart 1957-1984. In: Geschichte und Gesellschaft 11 (1985), S. 252-271. 
Böckenförde gegen Ende der sechziger Jahre geriet Hubers kompakte Deutung der Verfassungsgeschichte ernsthaft ins Wanken.

Otto Brunner gründete zusammen mit Werner Conze 1957 den Heidelberger »Arbeitskreis für moderne Sozialgeschichte ${ }^{57}$. Sein Werk »Land und Herrschaft « wurde methodisch wegweisend, vor allem nachdem Brunner in der vierten Auflage von 1959 das Wort »Volksgeschichte« regelmäßig durch »Strukturgeschichte« ersetzte, die dann in den sechziger Jahren auch noch zur »Sozialgeschichte « mutierte. Damit wurde er zu einer der Vaterfiguren der Historischen Sozialwissenschaft, die zu Beginn der siebziger Jahre historische Lehrstühle eroberte und zum neuen Leitsektor sich modern verstehender Geschichtsforschung avancierte.

Vor allem aufgrund des Engagements des Hartung-Schülers Gerhard Oestreich ergaben sich in der historisch ausgerichteten Verfassungsgeschichtsschreibung auch andere methodische Neuerungen, die an die verschüttete Tradition Otto Hintzes anknüpften und Verfassungsgeschichte wie Sozialgeschichte gleichermaßen inspirierten. Der von Oestreich initiierte Neudruck von Hintzes »Gesammelten Abhandlungen « Mitte der sechziger Jahre markierte den Beginn einer Renaissance des historiographischen Klassikers Hintze, die bis heute anhält ${ }^{58}$.

Seit den sechziger Jahren erfolgte eine generelle Trendwende in der Wissenschaftspolitik, die weithin auch einem Generationswechsel zu verdanken war. Für die Verfassungsgeschichte wiesen die Historikertage von 1967 und 1974 mit den Sektionen über das Verhältnis des Historikers zu Verfassung und Recht sowie Gegenstand und Begriffe der Verfassungsgeschichte neue Wege ${ }^{59}$. Mit dem Erscheinen der »Zeitschrift für Staatslehre, Öffentliches Recht und Verfassungsgeschichte«, »Der Staat«, seit 1961 und der Gründung der »Vereinigung für Verfassungsgeschichte 1977 schufen die führenden Verfassungshistoriker die Voraussetzungen für eine weitergehende Institutionalisierung ihrer Teildisziplin.

\section{Probleme und Perspektiven einer modernen Verfassungsgeschichtsschreibung seit 1970}

Eine zunehmende Institutionalisierung scheint das Kennzeichen der Verfassungsgeschichtsschreibung als Teildisziplin der Geschichts- und Rechtswissenschaften seit

$57 \mathrm{Zu}$ Conze: Thomas Etzemüller: Sozialgeschichte als politische Geschichte. Werner Conze und die Neuorientierung der westdeutschen Geschichtswissenschaft nach 1945, München 2001 (= Ordnungssysteme, 9).

58 Otto Hintze: Gesammelte Abhandlungen, hg. v. Gerhard Oestreich, Bd. 1: Staat und Verfassung. Gesammelte Abhandlungen zur allgemeinen Verfassungsgeschichte. Mit einer Einleitung v. Fritz Hartung. Bd. 2: Soziologie und Geschichte. Gesammelte Abhandlungen zur Soziologie, Politik und Theorie der Geschichte. Bd. 3: Regierung und Verwaltung. Gesammelte Abhandlungen zur Staats-, Rechts- und Sozialgeschichte Preußens, Göttingen 1962-1967.

59 Otto Brunner: Der Historiker und die Geschichte von Verfassung und Recht. In: Historische Zeitschrift 209 (1969), S. 1-16; Hermann Krause: Der Historiker und sein Verhältnis zur Geschichte von Verfassung und Recht. In: ebd., S. 17-26; Hans Thieme: Der Historiker und die Geschichte von Verfassung und Recht. In: ebd., S. 27-36. 
Beginn der sechziger Jahre zu sein. Die Verfassungshistoriographie hat sich, wie 1971 gefordert wurde, inhaltlich allerdings erst sukzessiv und mit Verzögerung zu einer »Sozial- und Organisationsgeschichte $«$ geöffnet $^{60}$. Damit ist sie die neuen Wege in der Geschichtswissenschaft allmählich mitgegangen, welche als erste Otto Brunner und Reinhart Koselleck beschritten hatten. Eine weitgehend stagnierende Entwicklung der Verfassungsgeschichte, wie sie bis dahin im Gegensatz zu den verbreiteten Modernisierungstrends anderer Wissenschaften festzustellen war, wurde seit den siebziger Jahren von einer neuen Dynamik abgelöst. Die vorher vielfach von Juristen und Historikern relativ isoliert betriebene Forschung verlief nun koordinierter. Zugleich erfolgte ein verstärkter Terraingewinn der Verfassungsgeschichte in den Geschichtswissenschaften selbst. Es bleibt abzuwarten, ob die zukünftige Verfassungsgeschichtsschreibung auch die neuen gesellschafts- und kulturgeschichtlichen Trends der Historiographie aufnehmen wird. Verfassungsgeschichtsschreibung hat sich tendenziell resistenter gegenüber historiographischen Neuansätzen erwiesen bzw. hat diese erst mit Zeitverzögerungen rezipiert. Ob sich eine »Verfassungsgeschichte in der Erweiterung «, wie sie Anfang der neunziger Jahre der Darmstädter Historiker Christof Dipper, anknüpfend an eine entsprechende Forderung Werner Conzes für die Sozialgeschichte der sechziger Jahre, gefordert hat ${ }^{61}$, tatsächlich durchsetzen kann, wird die wissenschaftshistorische Entwicklung der nahen Zukunft zeigen.

Wie steht es aber, um die Eingangsfrage aufzunehmen, mit den wechselseitigen Beziehungen von Politik und Wissenschaft, von Verfassungsrealität und Verfassungsgeschichtsschreibung? Unzweifelhaft hat die historische Entwicklung, haben die tiefen Zäsuren der deutschen Geschichte im 20. Jahrhundert Auswirkungen auf die verfassungsgeschichtliche Forschung gehabt. Ohne Frage hat die Staatsform des monarchischen Konstitutionalismus und der vorherrschende staatsrechtliche Positivismus die Verfassungsgeschichtsschreibung im Kaiserreich eher statisch werden lassen und systemstützend geprägt. Allein Otto Hintze hat mit seiner Innovationskraft die Systemgrenzen nicht sprengen wollen und auch nicht können. In den zwanziger Jahren inspirierte die neue Verfassungslage keineswegs $\mathrm{zu}$ einem demokratischen Umschwung der Verfassungsgeschichtsschreibung. Aber die Diskussion um einen erweiterten Verfassungsbegriff hat seit den späten zwanziger Jahren die staatliche und verfassungsgesetzliche Ausrichtung der historischen Forschung zu erschüttern vermocht. Nach 1933 hat dies dann in Verbindung mit antiliberalen Strömungen das aus

60 Hartwig Brandt: Verfassungsgeschichte als Sozial- und Organisationsgeschichte. In: Neue Politische Literatur 16 (1971), S. 242-255.

61 Christof Dipper: Sozialgeschichte und Verfassungsgeschichte. Zur Europäischen Verfassungsgeschichte aus der Sicht der Geschichtswissenschaft. In: Reiner Schulze (Hg.), Europäische Rechts- und Verfassungsgeschichte, Berlin 1991 (= Schriften zur Europäischen Rechts- und Verfassungsgeschichte, 3), S. 173-198, hier S. 178 f.; Werner Conze: Sozialgeschichte in der Erweiterung. In: Neue Politische Literatur 19 (1974), S. 501-508. Siehe auch Wolfgang Reinhard: Verfassungsgeschichte als Kulturgeschichte. Historische Grundlagen europäischer politischer Kulturen. In: Jahrbuch für Europäische Geschichte 1 (2000), S. 115-131; ders.: »Staat machen«. Verfassungsgeschichte als Kulturgeschichte. In: Jahrbuch des Historischen Kollegs 1998, S. 99-118. 
dem 19. Jahrhundert überkommene Bild der Verfassungsgeschichte nachhaltig in Frage gestellt. Nach 1945 befand sich die Verfassungsgeschichtsschreibung zunächst in der Defensive. Mit der Rückkehr der früheren Protagonisten in die Universitäten wandte sie sich indes entweder der Sozialgeschichte zu oder versuchte eine konservative Rettung der deutschen Geschichte. In den sechziger Jahren zeigte sich auch in der Verfassungsgeschichte ein allgemeiner Zug zur sozialgeschichtlichen Betrachtung, der sich in der Renaissance von Otto Hintze auswirkte.

Generell haben sich die verfassungshistorischen Zäsuren im ideengeschichtlichen Bereich der Wissenschaftsgeschichte am vergleichsweise deutlichsten bemerkbar gemacht - hier jedoch erst mit erheblicher zeitlicher Verzögerung. Die Anstöße des verfassungspolitischen Umbruchs von 1918/19 wirkten sich erst Ende der zwanziger Jahre im Staatsrecht und wiederum seit Mitte der dreißiger Jahre in der Verfassungsgeschichtsschreibung aus. Auch nach 1945 dauerte es bis in die sechziger Jahre, ehe eine sozialgeschichtliche Erweiterung Anhänger für die Verfassungsgeschichtsschreibung zu gewinnen vermochte. Deutlichere Zusammenhänge zwischen Politik und Wissenschaft zeigten sich dagegen im wissenschaftspolitischen Bereich. Hier sorgten die Zäsuren der Jahre 1933 und 1945 mit ihren ideologisch motivierten Personalrevirements für erhebliche Veränderungen in der Besetzung der verfassungshistorisch orientierten Lehrstühle.

In der Umbruchsituation des Jahres 1990 sei die Verfassungsgeschichte als »Depot kollektiver Erinnerungen für eine aufgewühlte Nation [...] aktuell wie nie zuvor« gewesen, resümierte der Rechtshistoriker Michael Stolleis nur ein Jahr später ${ }^{62}$. Inwieweit der deutsche Einigungsprozeß der Jahre 1989/90 sich auch längerfristig auf die Wissenschaftsgeschichte im allgemeinen und die Verfassungsgeschichtsschreibung im besonderen auswirken wird, dürfte gegenwärtig noch weitgehend offen sein.

62 Michael Stolleis: Neues zur Verfassungsgeschichte. In: Zeitschrift für neuere Rechtsgeschichte 23 (1991), S. 187-195, hier S. 187. 